\title{
System Applications of Holey Fibers
}

\author{
Ju Han Lee, David J. Richardson, Zulfadzli Yusoff, Walter Belardi, and Tanya M. Monro \\ Optoelectronics Research Centre, Southampton University, SO17 1BJ, UK \\ Tel: +442380592483 Fax: +442380593142 E-mail: jhl@orc.soton.ac.uk
}

\begin{abstract}
The wavelength-scale features in holey fibers lead to novel properties including endlessly single-mode guidance, high optical nonlinearity. The current HF technology for a wide range of device applications in optical communication systems is reviewed.
\end{abstract}

Recent progress in the design and fabrication of holey fiber (HF) has resulted in widespread interest in the use of such technology for a variety of photonics devices and applications [1]. Holey fibers (HFs) are a class of microstructured fiber which possess a solid core surrounded by a cladding region that is defined by a fine array of air holes that extend along the full fiber length (see Fig.1). HFs are typically made of a single material, usually pure silica, and guide light through a modified form of total internal reflection since volume average index in the core region of the fiber is greater than that of the surrounding microstructured cladding. Note that the hole diameter (d) and pitch ( $\Lambda=$ hole to hole spacing) which are the critical design parameters used to specify the structure of an HF are typically on the scale of the wavelength of light.

The strong wavelength dependence of the effectiveindex between the core and the cladding, and the possibility of large index contrasts between core and cladding in HF leads to a broader range of optical properties in these fibers than that realizable using conventional fiber fabrication approaches.
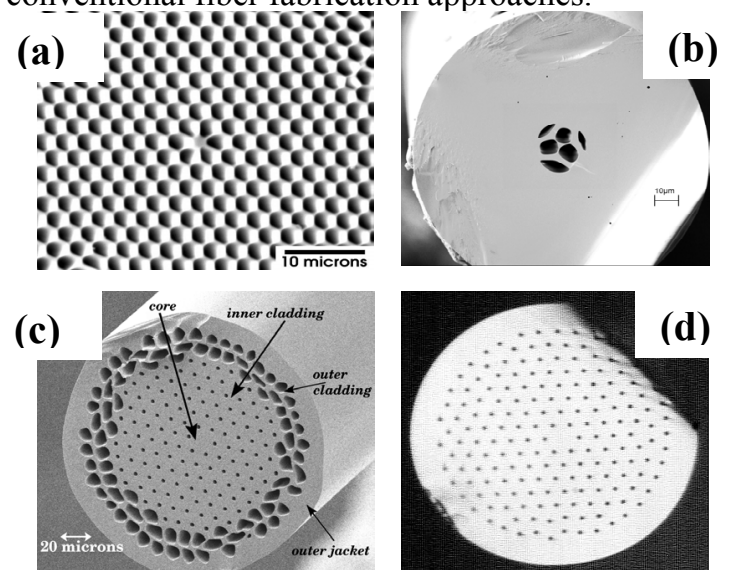

Fig.1. SEMs of various HFs fabricated at ORC: (a) small effective area silica HF, (b) high nonlinearity HF in Schott SF57 glass, (c) $\mathrm{Yb}^{3+}$ doped, air-clad, LMA HF, (d) LMA-HF.

Arguably, the most exciting possibility afforded by holey fiber technology is the opportunity to develop fibers with a very high optical nonlinearity per unit length $[2,3]$. Holey fibers with small-scale features
$($ small $\Lambda$ ) and a large air-filling fraction (large $\mathrm{d} / \Lambda$ ) can confine the guided mode tightly within the core, resulting in extremely small mode areas (see Fig.1a). One of the most promising applications of HFs is in the development of nonlinear optical devices for fiber-optic communication systems. HFs can have much higher nonlinearity per unit length than conventional fibers, and devices based on such fibers can thus be much shorter in length, and/or operate at lower power levels.

For example, we recently demonstrated a $2 \mathrm{R}$ data regeneration device based on a HF with an effective mode area of just $2.8 \mu \mathrm{m}^{2}$ and a nonlinearity $\gamma=31 \mathrm{~W}^{-}$ ${ }^{1} \mathrm{~km}^{-1}$ at $1.55 \mu \mathrm{m}$ [1]. The $2 \mathrm{R}$ regenerative operation was obtained by combining self-phase modulation and offset narrowband spectral filtering. Similar devices based on conventional fibers are typically of order $1 \mathrm{~km}$ in length whereas in our earliest experiments just $3.3 \mathrm{~m}$ of $\mathrm{HF}$ was needed for an operating power of $15 \mathrm{~W}$. We went on to use an $8.7 \mathrm{~m}$ long variant of this switch to provide an optical thresholding function within an optical code division multiple access (OCDMA) system [4]. A schematic of the thresholder is shown in Fig.2a.
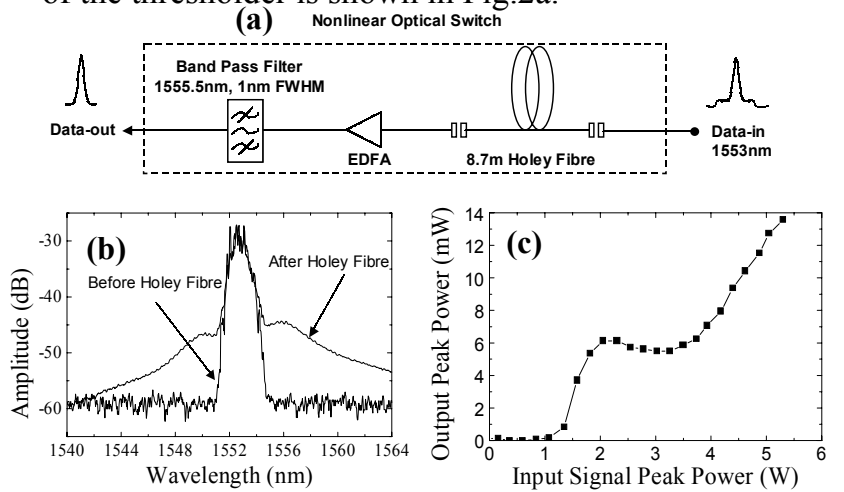

Fig.2. (a) The schematic of the nonlinear optical thresholder, (b) spectra of pulse before and after HF, (c) power transmitted through the full system including the offset narrowband filter.

In Fig. $2 b$ we show the spectrum of 2.5 ps soliton pulses both prior and after propagation through the highly nonlinear HF. As can be seen from Fig.2b new spectral components are generated at both red and blue shifted frequencies relative to the incoming spectrum. In Fig.2c we show the pulse power transmission characteristic as a function of incident pulse peak power. The S-shaped characteristic is suitable for optical thresholding applications. We used such a switch prior to our receiver to eliminate the low-level pedestal components arising from the 
matched filtering of a coded bit and obtained a significant improvement in the overall system performance.

Such fibers also offer reduced length/power requirements for nonlinear devices based on other nonlinear effects such as the Brillouin and Raman effects. For example, we recently demonstrated a $\sim 70 \mathrm{~m}$ long, fiber laser pumped Raman amplifier [5]. The experimental setup is shown in Fig.3a.

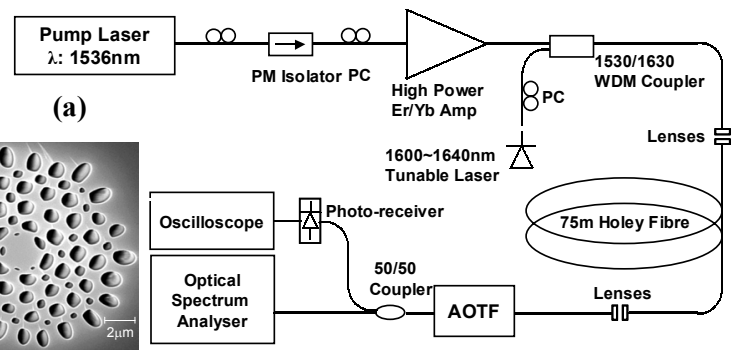

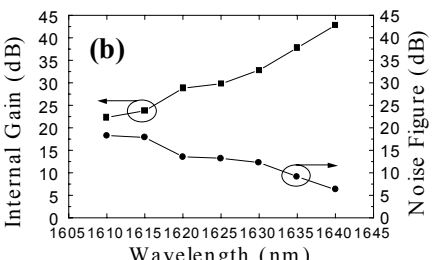

Wavelength $(\mathrm{nm})$

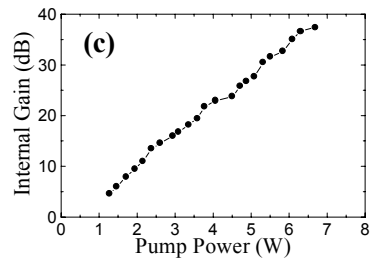

Pump Power (W)
Fig.3. (a) The schematic of our HF amplifier (AOTF-acousto-optic tuneable filter); Inset: SEM image of the HF used. (b) Wavelength dependence of gain, (c) gain efficiency curve at a wavelength of $1635 \mathrm{~nm}$.

The amplifier was pumped using a pulsed fiber laser and provided high gains of up to $43 \mathrm{~dB}$ in the $\mathrm{L}^{+}$ communications band (see Fig.3b) for pulse peak powers of order $7 \mathrm{~W}$ (see Fig.3c). More recently, we constructed a continuous wave Raman laser pumped at $1080 \mathrm{~nm}$ using a high-power, cladding-pumped $\mathrm{Yb}^{3+}$-doped fiber laser [6]. The laser had a $\mathrm{CW}$ threshold of $5 \mathrm{~W}$, and slope efficiency of $\sim 70 \%$. Of particular note, was the $\mathrm{CW}$ power density at the facet which ran at a power level of $0.2 \mathrm{GW} / \mathrm{cm}^{2}$, demonstrating that such small-core, single-material HFs can exhibit a good resilience to optically induced damage.

Wavelength conversion is another important function required within current high-capacity WDM systems. We recently demonstrated that HF technology can be applied to multiple wavelength conversion over a $\sim 20 \mathrm{~nm}$ bandwidth at a data rate of $10 \mathrm{Gbit} / \mathrm{s}$ using a combination of XPM in a short length of HF, and narrowband spectral filtering [7]. The experimental setup is shown in Fig.4a. XPM between the control signal and the $\mathrm{CW}$ beams results in chirping of the $\mathrm{CW}$ laser beam where these beams overlap temporally within the fiber. This frequency chirping can then be converted to a frequency converted signal by passing the signal through a narrowband filter which serves to eliminate the residual unchirped $\mathrm{CW}$ signal as well as to select one of the two XPM-induced side bands. The pulsewidths of the converted pulses were observed to be almost constant at $\sim 5.8$ ps over a wavelength range of $\sim 20 \mathrm{~nm}$ (see Fig.4c).

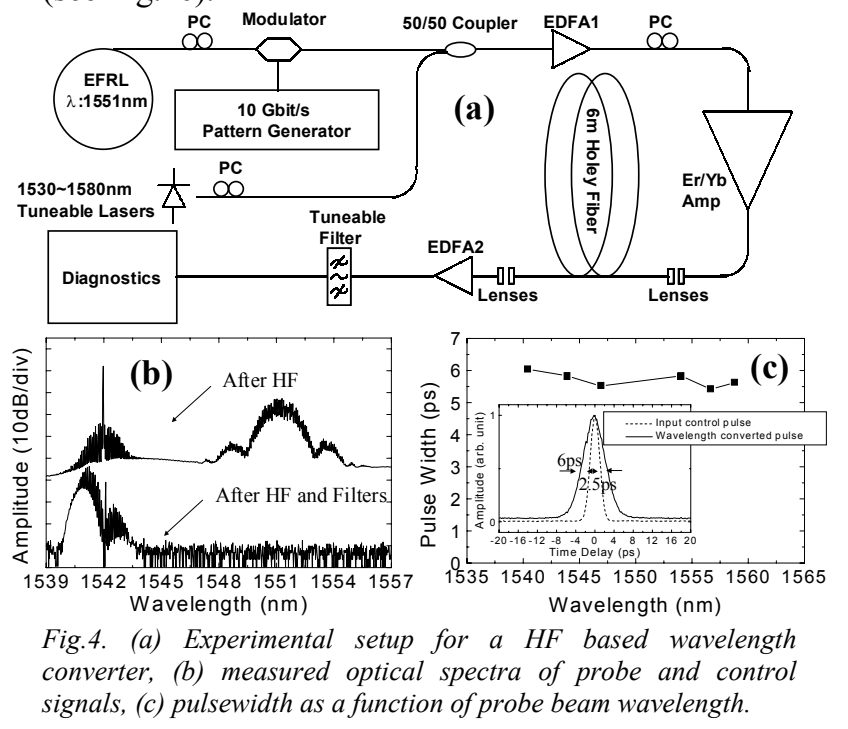

Apart from the works mentioned above, many other research groups have demonstrated a variety of HF based nonlinear devices such as nonlinear optical loop mirrors [8], Brillouin lasers [9] and so on. Note that these previously described device demonstrations have used silica based holey fibers but that further significant increases in fiber nonlinearity should be achievable using fibers made of other glasses, such as the Chalcogenides, which have around two orders of magnitude higher nonlinear optical coefficient than silica. Indeed, recently we produced the first results in this direction and demonstrated a HF in SF57 lead glass with $\gamma=550 \mathrm{~W}^{-1} \mathrm{~km}^{-1}$, about 500 times more nonlinear than conventional SMF28 fiber [10]. Note that polymer HFs have also now been produced [11].

In conclusion, holey fiber technology has advanced now to the point that $\mathrm{km}$-lengths of robust coated fiber can be produced with losses below $1 \mathrm{~dB} / \mathrm{km}$ [12], with a broad range of unique and useful optical properties. Such fibers have the potential to enable a wide range of practical nonlinear optical devices for a wide range of applications areas both within fiber optic communications and beyond.

[1] P. Petropoulos et al., Optics Lett., 26, p.1233 (2001).

[2] N.G.R.Broderick et al., Optics Lett., 24, p.1395, (1999).

[3] K.P. Hanse et al., OFC 2002, Postdeadline FA9-1, (2002).

[4] J.H. Lee et al., Photon. Technol. Leet., 14, p.876, (2002).

[5] Z. Yusoff et al., Optics Lett., 27, p.424 (2002).

[6] J. Nilsson et al., OFC 2002, Paper WR6, (2002).

[7] J.H. Lee et al., CLEO'2002, Postdeadline (2002)

[8] J.E. Sharping et al., Photon. Technol. Lett.,14, p.77 (2002).

[9] J.H. Lee et al., Optics Lett., 27, p.927, (2002).

[10] T.M. Monro et al., OFC'2002, Postdeadline FA1-1, (2002).

[11] M. A. van Eijkelenborg et al., Opt. Express, 9, p.319 (2001).

[12] K. Tajima et al., OFC'2002, p.523, (2002). 